\title{
Primal-dual for classification with rejection (PD-CR): a novel method for classification and feature selection-an application in metabolomics studies
}

\author{
David Chardin ${ }^{1,2}$, Olivier Humbert ${ }^{1,2}$, Caroline Bailleux ${ }^{1,4}$, Fanny Burel-Vandenbos ${ }^{5}$, Valerie Rigau ${ }^{6,7}$, \\ Thierry Pourcher ${ }^{1}$ and Michel Barlaud ${ }^{3 *}$
}

${ }^{*}$ Correspondence:

barlaud@i3s.unice.fr

${ }^{3}$ Laboratoire d'Informatique,

Signaux et Systèmes de

Sophia Antipolis (I3S),

Université Côte d'Azur

(UCA), Centre de Recherche

Scientifique (CNRS), Sophia

Antipolis, France

Full list of author information is available at the end of the article

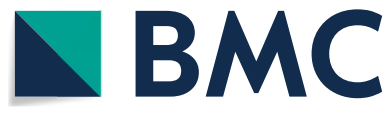

(c) The Author(s), 2021. Open Access This article is licensed under a Creative Commons Attribution 4.0 International License, which permits use, sharing, adaptation, distribution and reproduction in any medium or format, as long as you give appropriate credit to the original author(s) and the source, provide a link to the Creative Commons licence, and indicate if changes were made. The images or other third party material in this article are included in the article's Creative Commons licence, unless indicated otherwise in a credit line to the material. If material is not included in the article's Creative Commons licence and your intended use is not permitted by statutory regulation or exceeds the permitted use, you will need to obtain permission directly from the copyright holder. To view a copy of this licence, visit http:// creativecommons.org/licenses/by/4.0/. The Creative Commons Public Domain Dedication waiver (http://creativecommons.org/publi cdomain/zero/1.0/) applies to the data made available in this article, unless otherwise stated in a credit line to the data.

Results: PD-CR was more accurate than PLS-DA, Random Forests and SVM for classification using the 2 metabolomics datasets. It also selected biologically relevant metabolites. PD-CR has the advantage of providing a confidence score for each prediction, which can be used to perform classification with rejection. This substantially reduces the False Discovery Rate.

Conclusion: PD-CR is an accurate method for classification of metabolomics datasets which can outperform PLS-DA, Random Forests and SVM while selecting biologically relevant features. Furthermore the confidence score provided with PD-CR can be used to perform classification with rejection and reduce the false discovery rate. 


\section{Introduction}

Among the different omics fields, metabolomics is the most recent and provides new insights for a global study of biological systems. Metabolomics is a rapidly growing and promising field of research in biology and healthcare. Metabolomics approaches are based on the determination of the levels of different small molecules or metabolites in biological samples (tissue, cells, serum, urine...). Interestingly, ever since the early metabolomics studies, supervised classification methods have been used for the analysis of the related datasets. One of the initial aims of metabolomic studies was to establish useful biomarkers, indicative of specific physiological states or aberrations. The challenge now is to understand the mechanisms by which changes in the metabolome are implicated in different phenotypic outcomes in a complex systems biology approach [1,2].

Most metabolomics studies generate complex multivariate datasets including varying correlations between features and systematic noise. Therefore, multivariate data analysis methods are needed to explore these datasets. One of the most frequently used methods for metabolomics analyses is Partial Least Squares-Discriminant Analysis (PLS-DA) [3, 4].

PLS-DA is a chemometric technique used to optimize separation between different classes of samples, which is accomplished by linking two data matrices: $\mathrm{X}$ (raw metabolomic data) and $\mathrm{Y}$ (class membership). It has the advantage of handling highly collinear and noisy data. Yet, it has some drawbacks and needs to be handled with caution. Indeed it has been reported that PLS-DA can: 1. Lead to over-fitting when the number of variables significantly exceeds the number of samples. Indeed, in this setting, the model is likely to lead to accurate classification by chance, based on irrelevant features [5]; 2. Have difficulties when few variables are responsible for the separation between two or more classes and, therefore, require a larger number of variables to achieve a good prediction accuracy [6]; and finally, 3. Lead to an over-optimistic understanding of the separation between two or more classes [7].

Continuous effort is being made to provide new statistical tools to tackle these drawbacks [8]. Some authors use Random Forests [9] as an alternative to PLS-DA for metabolomics studies [10]. Random Forests are based on the bagging algorithm and use an Ensemble Learning technique. Random Forests create a large number of decision trees and combine their outputs. Yet, Random Forests have significant drawbacks. For instance, they tend to over-fit when using noisy datasets. Furthermore, the main disadvantage of Random Forests is their complexity. Indeed, they are much harder and time-consuming to construct, require more computational resources and are less intuitive than decision trees. Furthermore this complexity significantly hampers their interpretability. Support Vector Machines (SVM) are another option [11, 12] but have similar drawbacks as Random Forests and are particularly consuming in computational resources.

Mathematics I3S partner has recently introduced a new tailored, constrained primal-dual method for supervised classification and feature selection [13]. This method has the significant advantage of providing a trustworthy confidence index with each prediction, which we use to define a new classifier with rejection. This is particularly useful in the context of clinical decision making as it diminishes the number of false positive and false negative results. 
Moreover, we believe this method out-performs other methods in terms of accuracy and feature selection.

Although there are many machine learning methods for feature selection such as LASSO [14, 15], Discriminant analysis [16], Proximal methods [17, 18] and Boosting [19, 20], here we compare our novel Primal-Dual method for Classification with Rejection (PD-CR) to the state of the art PLS-DA and Random Forests and SVM classification methods frequently used in metabolomics studies.

\section{Methods}

\section{Mathematical background}

\section{Robust classification and regression using $\ell_{1}$ centers}

Mathematically, classification problems can be described as follows:

Let $X$ be the $m \times d$ data matrix made of $m$ line samples $x_{1}, \ldots, x_{m}$ that belong to the $d$-dimensional space of features.

Let $Y \in\{0,1\}^{m \times k}$ be the matrix of labels where $k \geq 2$ is the number of clusters. Each line of $Y$ has exactly one nonzero element equal to one, $y_{i j}=1$ indicating that the sample $x_{i}$ belongs to the $j$-th cluster. Projecting the data in lower dimension is crucial to be able to separate them accurately.

Let $W$ be the $d \times k$ projection matrix, where $k \ll d$. (Note that the dimension of the projection space is equal to the number of clusters.)

The goal of the supervised classification method is to find the best possible values for the projection matrix $W$.

Sparse learning based methods have received a lot of attention in the last decade because of their high level of performance. The basic idea is to use a sparse regularizer that forces some coefficients to be zero. To achieve feature selection, the Least Absolute Shrinkage and Selection Operator (LASSO) formulation [14, 21-25] adds an $\ell_{1}$ penalty term to the classification cost. An accurate criterion is based on the sum of the square difference (used in k-means [26]) and can be cast as follows:

$$
\|Y \mu-X W\|_{F}^{2}=\sum_{j=1}^{k} \sum_{l \in C_{j}}\left\|(X W)(l,:)-\mu_{j}\right\|_{2}^{2},
$$

where $C_{j} \subset\{1, \ldots, m\}$ denotes the $j$-th class, and where the row vector $\mu_{j}$ is the centroid of this class. Therefore, the matrix of centers $\mu$ is a square matrix of order $k$. It is well known that the Frobenius norm is sensitive to outliers. To address this, we have improved the approach by replacing the Frobenius norm by the $\ell_{1}$ norm of the loss term as follows:

$$
\|Y \mu-X W\|_{1}=\sum_{j=1}^{k} \sum_{l \in C_{j}}\left\|(X W)(l,:)-\mu_{j}\right\|_{1} .
$$

where $C_{j} \subset\{1, \ldots, m\}$ denotes the $j$-th cluster, and where $\mu_{j}:=\mu(j,:)$ is the $j$-th line of $\mu$. In our method, we simultaneously optimize $(W, \mu)$, adding some ad hoc penalty to break homogeneity and avoid the trivial solution $(W, \mu)=(0,0)$. 
Using both the projection $W$ and the centers $\mu$ learnt during the training step, a new query $x$ in the test set (a dimension $d$ row vector) is classified according to the following rule: it belongs to the cluster number $j^{*}$ if and only if

$$
j^{*} \in \arg \min _{j=1, \ldots, k}\left\|\mu_{j}-x W\right\|_{1} .
$$

\section{Primal-dual scheme, constrained formulation}

To handle features with a high correlation, we consider a convex constrained supervised classification problem. However the drawback of the term $\|Y \mu-X W\|_{1}$ is that it enforces equality of the two matrices out of a sparse set: hence it tunes the parameters to enforce a perfect matching of the training data. We replace the 1-norm with the robust "Huber function" [13]. If $h_{\delta}(t)=t^{2} /(2 \delta)$ for $|t| \leq \delta$ and $|t|-\delta / 2$ for $|t| \geq \delta$.

We obtain the following criterion

$$
\min _{(W, \mu)} h_{\delta}(Y \mu-X W)+\frac{\rho}{2}\left\|I_{k}-\mu\right\|_{F}^{2} \text { s.t. } \quad\|W\|_{1} \leq \eta .
$$

We can tune a primal-dual method to solve this problem with Algorithm 1 (See [13] and [27] for details)

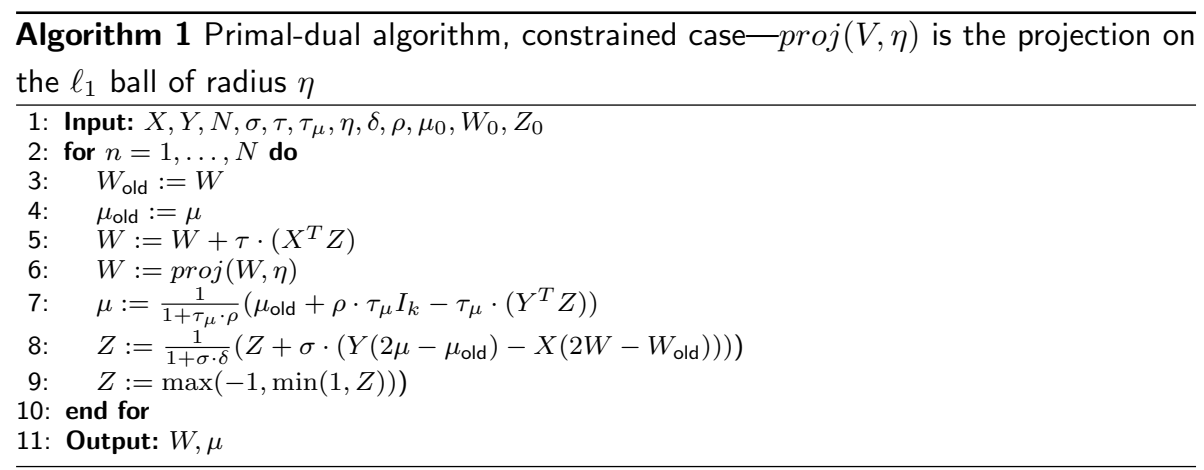

\section{Classification with rejection using a confidence Score for the Prediction (CSP)}

False positive (FP) and false negative (FN) results are an important issue for diagnostic tools in medicine. One way to diminish the number of FP and FN results is to use classification with rejection $[19,28]$ for which classifiers are allowed to report "I don't know". This type of classification enables the incorporation of doubt in the results if the observation $\mathrm{x}$ is too hard to classify. Here, we propose to use a confidence score for the prediction (CSP) to devise a classifier with rejection.

In our analysis we only had two clusters with centers $\mu_{1}$ and $\mu_{2}$ Lets recall that the predicted label $j^{*}$ of a sample $\mathrm{x}$ is given by

$$
j^{*} \in \arg \min _{j=1, \ldots, 2}\left\|\mu_{j}-x W\right\|_{1} .
$$


We can compute the distances of sample $\mathrm{x}$ to the two centroids, respectively. $d_{1}=\left\|\mu_{1}-x W\right\|_{1}$ and $d_{2}=\left\|\mu_{2}-x W\right\|_{1}$ and we propose a confidence indicator for sample $\mathrm{x}$ as follows:

$$
\rho(x)=\frac{d_{1}-d_{2}}{d_{1}+d_{2}}
$$

Thus, the CSP $\rho(x)$ is a value ranging from -1 to 1 . The closer the CSP $\rho(x)$ is to +1 or -1 depending on the predicted class, the higher the confidence for the prediction will be.

Thus if $\epsilon$ is a given threshold parameter, we can perform classification with rejection by rejecting binary classification for samples with an absolute value of CSP $\rho(x)$ under this threshold. The labels will then be predicted as follows:

$$
\text { Label }= \begin{cases}-1 & \text { if } \rho(x)<-\epsilon \\ 0 & \text { if }-\epsilon<\rho(x)<\epsilon \\ 1 & \text { if } \rho(x)>\epsilon\end{cases}
$$

We can then study the False Discovery Rate (FDR) $F D R=F P+F N$ as a function of parameter $\epsilon$.

\section{Comparison to PLS-DA, Random Forests and SVM using 2 datasets}

To compare PD-CR to the standard PLS-DA, Random Forests and SVM classification methods in terms of accuracy and feature selection, we tested the four methods on two metabolomic datasets named "BRAIN" and "LUNG". Accuracies and feature selection for each method were obtained using 4 fold-cross validation with varying random seeds. We also provide the results with a new version of PD-CR minimizing the $\ell 2$ norm PD-CR $\ell 2$ (See Algorithm 6 https : //arxiv.org/pdf/1902.01600.pdf).

\section{LUNG dataset}

The LUNG dataset was provided by Mathe et al. This dataset includes metabolomics data concerning urine samples from 469 Non-Small Cell Lung Cancer (NSCLC) patients prior to treatment and 536 controls collected from 1998 to 2007 in seven hospitals and in the Department of Motor Vehicles (DMV) from the greater Baltimore, Maryland area. Urine samples were analyzed using an unbiased metabolomics LC-MS/MS approach. This dataset is available from the MetaboLights database (study identifier MTBLS28)

Mathe et al. used Random Forests to classify patients as lung cancer patients or controls [10]. The aim was to create a new screening test for lung cancer, based on metabolomics data from urine. Lung cancer is one of the most common cancers and it is well established that early diagnosis is essential for treatment. An efficient screening method based on urinary metabolomics would be of great benefit.

\section{BRAIN dataset}

The BRAIN dataset was obtained from a metabolomic study performed by our biological team (TIRO) on frozen samples of glial tumors. The samples were provided by the university hospitals of Nice and Montpellier (France). Metabolite extracts were prepared and analyzed in the TIRO laboratory (Nice, France). With this dataset, the goal was to create a model that accurately discriminated between mutated isocitrate dehydrogenase 
(IDH) and IDH wild-type glial tumors. This mutation is a key component of the World Health Organization classification of glial tumors [29]. The mutational status is usually assessed by IDH1 (R132H)-specific (H09) immunohistochemistry. Yet this technique can lead to False-Negative results [30], which can only be identified by sequencing. An accurate metabolomic based test, able to assess the IDH mutational status, could be a promising solution to this problem.

These samples were retrospectively collected from two declared biobanks from the Central Pathology Laboratory of the Hospital of Nice and from the Center of Biological Resources of Montpellier (Plateforme CRB-CHUM). Consent or non-opposition was verified for every participant. For every participant, the IDH mutational status was assessed using immunohistochemistry and pyrosequencing for immunonegative cases.

Samples of brain tumors were analyzed using Liquid Chromatography coupled to tandem Mass Spectrometry (LC-MS/MS) in an unbiased metabolomics approach, as performed in a previous metabolomics study $[\mathrm{xxx}]$.

The details of the analysis are available in Additional file 1.

\section{Data Filtering and Pre-processing}

Our laboratory performed the LC-MS/MS analysis for the BRAIN dataset. Therefore, we could apply different levels of filtering on this dataset. After processing of the raw data using MZmine 2.39 software, two types of filtering were applied to the BRAIN dataset, minimal and maximal filtering. The minimal filtering only removed metabolites for which a spike was detected in less than 10 percent of the samples. The maximal filtering removed all unidentified metabolites as well as metabolites that did not have an isotopic pattern. This filtering method is frequently used for metabolomic studies and diminishes the number of noisy features in the dataset. Furthermore, it diminishes the time necessary for data processing because it diminishes the data volume. Unfortunately, any filtering will necessarily come with a high risk of removing some relevant features which is also the case with this filtering method. Using the two BRAIN datasets, we aimed to assess how the filtering affected the results of the different classification methods. The LUNG dataset was used as it was published, without additional normalization or filtering.

\section{Comparison to other methods}

Before comparison, the data were pre-processed as follows:

(i) Log-transformation for the following benefits: Reducing heteroscedasticity and thus the bias on regression and transforming multiplicative noise into additive noise,

(ii) Mean centering and scaling [31].

PD-CR [13] was compared to PLS-DA [32], Random Forests (with 100 and 400 trees) [9] and SVM using the sklearn python package.

Additionally, we evaluated the impact of the use of the Huber loss in PD-CR compared to the use of the $\ell 2$ loss.

Parameters $\sigma, \tau, \delta$ and $\rho$ were set according to results obtained using various datasets in an initial step [13] and were not further tuned. Parameter $\eta$, which affects the feature 
Table 1 Overview of the datasets

\begin{tabular}{lccl}
\hline Dataset & No. of samples & No. of features & Sample type \\
\hline LUNG & 1005 & 2944 & Urine \\
BRAIN & 88 & 25,286 & Glial tumor tissue
\end{tabular}

Table 2 LUNG dataset: mean accuracy using 3 seeds and 4-fold cross validation: comparison with PLS-DA, Random forest and Best SVM

\begin{tabular}{lllllll}
\hline LUNG & PD-CR & PD-CR $\ell_{2}$ & PLS-DA & RF (100 trees) & RF (400 trees) & SVM \\
\hline Accuracy \% & 79.44 & 78.3 & 76.56 & 71.31 & 72.44 & 76.25 \\
AUC & 79.97 & - & 74.05 & 73.38 & 74.50 & 76.64 \\
Time $(\mathrm{s})$ & 0.11 & 0.11 & 0.09 & 0.89 & 3.47 & 85.6 \\
\hline
\end{tabular}

selection step was manually tuned to fit the number of features in the datasets and to maximize accuracy after cross validation.

We computed the accuracy of the 4 classification methods for the two metabolomics datasets using 4-fold cross-validation (Script "PD-CR vs PLS-DA, RF and SVM" on https://github.com/tirolab/PD-CR). The selected metabolites were analyzed and compared between methods for the metabolomics datasets.

For PD-CR, we plotted the histograms of the CSP $\rho(x)$ and the probability distribution function $(\mathrm{PDF})$ as well as the False Discovery Rate $(\mathrm{FDR}=(\mathrm{FP}+\mathrm{FN}) /$ total $)$ and the rate of rejected samples (RRS $=$ rejected samples/total samples) depending on epsilon (the CSP threshold) (Script "rhoComputing" on https://github.com/tirolab/PD-CR).

\section{Results}

The characteristics of the two metabolomics datasets are presented in Table 1.

The LUNG dataset included a large number of patients (a little over 1,000) with an equivalent number of features (a little under 3,000) and the BRAIN dataset included a smaller number of patients (88) with a much higher number of features. While obtaining metabolomics data concerning as many patients as there are in the LUNG dataset is remarkable, the number of patients in the BRAIN dataset is closer to the number of patients in most metabolomics studies.

\section{LUNG}

As shown in Table 2, PD-CR outperformed PD-CR $\ell_{2}$, PLS-DA, Random Forests (400 trees) and SVM by $1.1 \%, 2.8 \%, 7 \%$ and $3.1 \%$ respectively.

Even though an accuracy of $79.44 \%$ may be high enough to consider using our PD-CR method and urinary metabolomics for the screening of lung cancer, Fig. 1 shows that the accuracy may be even higher if the CSP is taken into account and if it is used to perform classification with rejection. Indeed, in Fig. 1 the top left shows the histogram of the CSP and the top right the kernel probability distribution function (PDF). We can see that healthy controls and cancer patients are predicted with an equally high confidence. On the bottom left the False Discovery 

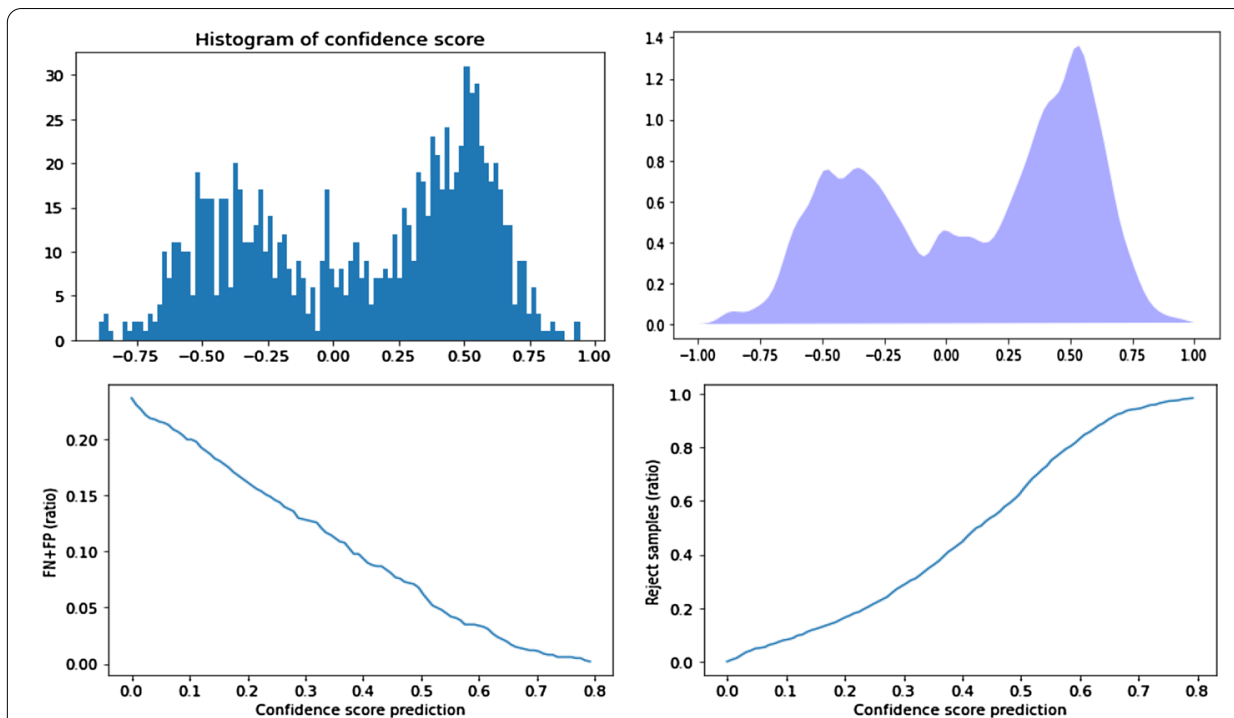

Fig. 1 Distribution of the confidence score for the prediction (CSP) on the Lung dataset and impact of using CSP for classification with rejection on the false discovery rate (FDR). From Left to right and top to bottom: Histogram of the CSP, Kernel density estimation; FDR as a function of CSP after classification with rejection, rate of rejected samples as a function of CSP after classification with rejection. As expected for a pertinent confidence score, the FDR diminishes when using a higher CSP threshold for classification with rejection

Table 3 Top 10 features selected by random forests, PLS-DA, PD-CR and SVM in the LUNG dataset

\begin{tabular}{|c|c|c|c|}
\hline $\mathrm{RF}$ & PLS-DA & PD-CR & SVM \\
\hline MZ 264.1215224 & MZ 264.1215224 & MZ 264.1215224 & MZ 264.1215224 \\
\hline MZ 656.2017529 & MZ 126.9069343 & MZ 308.0984878 & MZ 308.0984878 \\
\hline MZ 441.1613664 & MZ 170.0605916 & MZ 126.9069343 & MZ 247.0970455 \\
\hline MZ 584.2670695 & MZ 613.3595637 & MZ 613.3595637 & MZ 613.3595637 \\
\hline MZ 247.0970455 & MZ 243.1004849 & MZ 243.1004849 & MZ 615.0353192 \\
\hline MZ 486.2571336 & MZ 486.2571336 & MZ 247.0970455 & MZ 372.9232556 \\
\hline MZ 308.0984878 & MZ 308.0984878 & MZ 332.0963401 & MZ 441.1613664 \\
\hline MZ 204.1345526 & MZ 561.3432022 & MZ 441.1613664 & MZ 370.0525988 \\
\hline MZ 247.1384435 & MZ 94.06574518 & MZ 94.06574518 & MZ 423.0084949 \\
\hline MZ 447.10803 & MZ 269.1280232 & MZ 561.3432022 & MZ 332.0963401 \\
\hline
\end{tabular}

Rate $(F D R=(F P+F N) /$ total samples $)$ decreases as the confidence score threshold increases, but as shown in the bottom right, the rate of rejected samples $(R R S=$ rejected samples/total samples $)$ increases.

As shown in Table 3, PD-CR selected "MZ 264.1215224" for a molecular ion at $\mathrm{m} / \mathrm{z}$ 264.1215224 and "MZ 308.0984878" for a molecular ion at $\mathrm{m} / \mathrm{z} 308.0984878$ as the top two features.

These features "MZ 264.1215224" and "MZ 308.0984878" most likely correspond to creatine riboside (expected $\mathrm{m} / \mathrm{z}$ value in the positive mode: 264.1190; mass error: $10 \mathrm{ppm}$ ) and $\mathrm{N}$-acetylneuraminic acid (expected $\mathrm{m} / \mathrm{z}$ value in the negative mode: 308.0987; mass error: $1 \mathrm{ppm}$ ), respectively. These two metabolites were described by Mathé et al. [10] as the two most important metabolites to discriminate between lung 


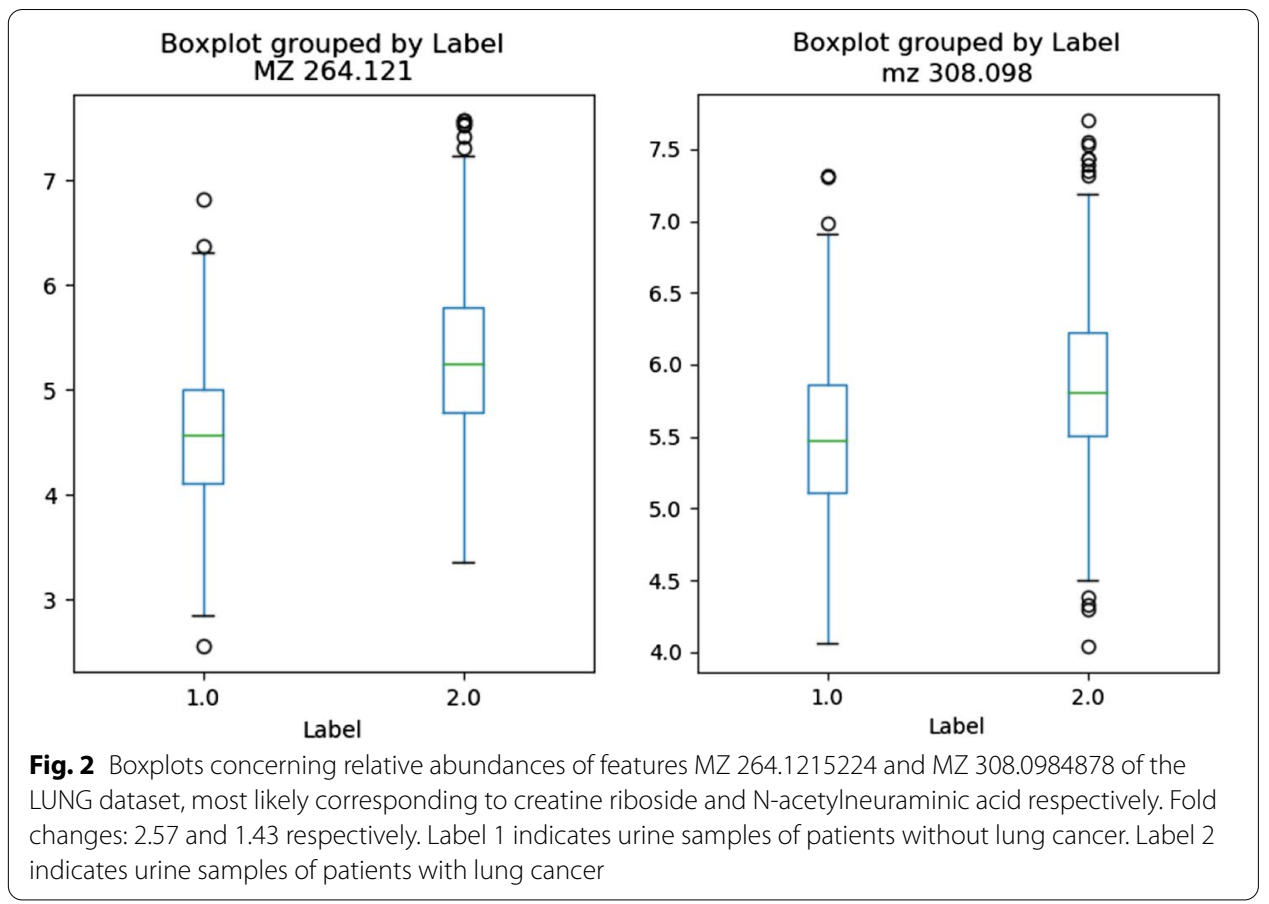

Table 4 BRAIN dataset Accuracy using 3 seeds and 4-fold cross validation: comparison with PLS-DA, Random Forest and best SVM

\begin{tabular}{lllllll}
\hline BRAIN & PD-CR & PD-CR $\ell_{2}$ & PLS-DA & RF (100 trees) & RF (400 trees) & SVM \\
\hline Accuracy $\%$ & 92.04 & 90.9 & 84.09 & 88.63 & 89.39 & 87.78 \\
AUC & 92.08 & - & 84.33 & 88.70 & 89.02 & 88.53 \\
\hline
\end{tabular}

cancer patients and healthy individuals using Random Forests on metabolomic data from urine samples. Indeed, these two metabolites were significantly higher in the urines of lung cancer patients, as shown in Fig. 2.

\section{BRAIN}

Minimally filtered dataset

As shown in Table 4, PD-CR outperformed PD-CR $\ell_{2}$, PLS-DA, Random Forests (400 trees) and SVM by $1.1 \%, 7.7 \%, 2.7 \%$ and $4.3 \%$, respectively for the BRAIN dataset. For this high dimensional dataset, the number of features $(25,286)$ significantly exceeded the number of samples (88) giving a significant drop in the PLS-DA accuracy.

Furthermore, as shown in Fig. 3 the accuracy obtained with PD-CR could be further improved by using the CSP to perform classification with rejection. Indeed, most of the samples were classified with a high CSP and if we apply a CSP threshold $\epsilon$ of 0.45 , the FDR drops to 0 while only rejecting $10 \%$ of the samples. This shows that all the missclassified samples had a low CSP.

As shown in Table 5, most of the top features selected with the 3 methods correspond to different isotopes and adducts of 2-hydroxyglutarate. Indeed, POS MZ131.0342, POS_MZ132.0375 and POS_MZ133.0384 all correspond to the 


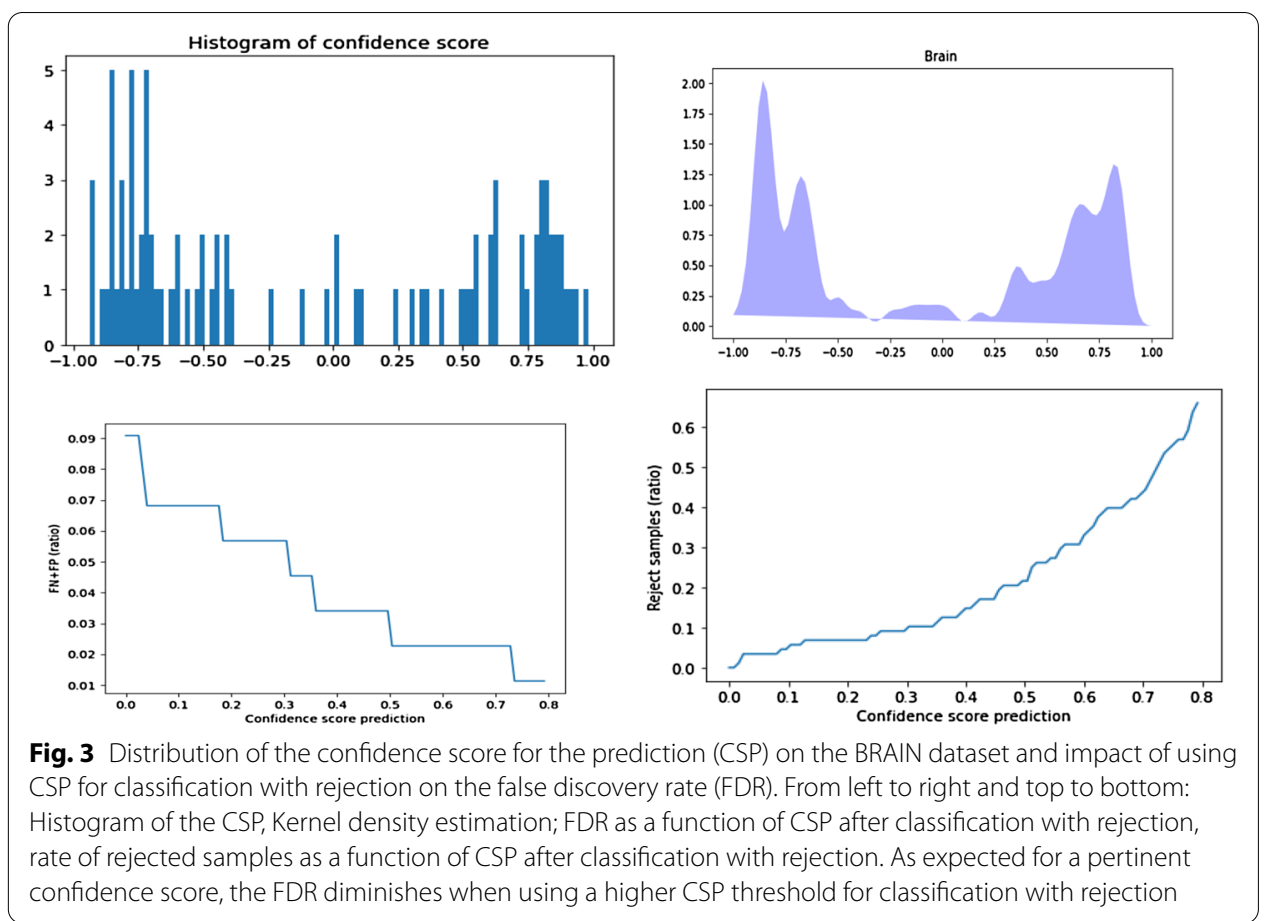

Table 5 Top 10 features selected by random forests, PLS-DA, PD-CR and SVM on the BRAIN dataset with 25,286 features

\begin{tabular}{llll}
\hline Random forests & PLS-DA & PD-CR & SVM \\
\hline NEG_MZ147.0867 & POS_MZ131.0342 & POS_MZ131.0342 & POS_MZ131.0342 \\
POS_MZ133.0384 & POS_MZ132.0375 & POS_MZ132.0375 & POS_MZ132.0375 \\
POS_MZ166.0713 & POS_MZ166.0713 & POS_MZ243.9903 & POS_MZ166.0713 \\
POS_MZ228.0182 & NEG_MZ147.0288 & POS_MZ166.0712 & NEG_MZ147.0288 \\
POS_MZ132.5234 & NEG_MZ148.0321 & NEG_MZ147.0288 & NEG_MZ148.0321 \\
POS_MZ173.0306 & NEG_MZ149.0329 & NEG_MZ148.0321 & POS_MZ171.0265 \\
POS_MZ219.0082 & POS_MZ171.0265 & POS_MZ123.5181 & POS_MZ132.0375 \\
NEG_MZ215.0168 & POS_MZ132.0375 & POS_MZ171.0265 & POS_MZ247.9616 \\
POS_MZ171.0265 & POS_MZ243.9903 & NEG_MZ149.0329 & POS_MZ243.9903 \\
POS_MZ319.0510 & POS_MZ123.5181 & POS_MZ133.0384 & NEG_MZ149.0329 \\
\hline
\end{tabular}

$[\mathrm{M}+\mathrm{H}-\mathrm{H} 2 \mathrm{O}$ adduct $]+$ of 2-hydroxyglutarate with $\mathrm{C} 12$, and two $\mathrm{C} 13$ isotopes respectively. NEG_MZ147.0288, NEG_MZ148.0321 and NEG_MZ149.0329 correspond to the $[\mathrm{M}-\mathrm{H}]$ - adduct with $\mathrm{C} 12$, and two C13 isotopes respectively. POS_MZ166.0713 corresponds to a $[\mathrm{M}+\mathrm{NH} 4]+$ adduct. POS_MZ171.02645 corresponds to the $[\mathrm{M}+\mathrm{Na}]+$ adduct. POS_MZ243.9903 had the same retention time and chromatographic profile as POS_MZ131.0342, suggesting that it was an unknown fragment or adduct of 2-hydroxyglutarate.

2-Hydroxyglutarate is a well-known oncometabolite produced in high quantities by mutated IDH1/2 in gliomas [33]. It is therefore expected that this compound will have a high weight when classifying mutated vs wild-type gliomas as it should be significantly increased in IDH mutated gliomas (as shown in Fig. 4). 


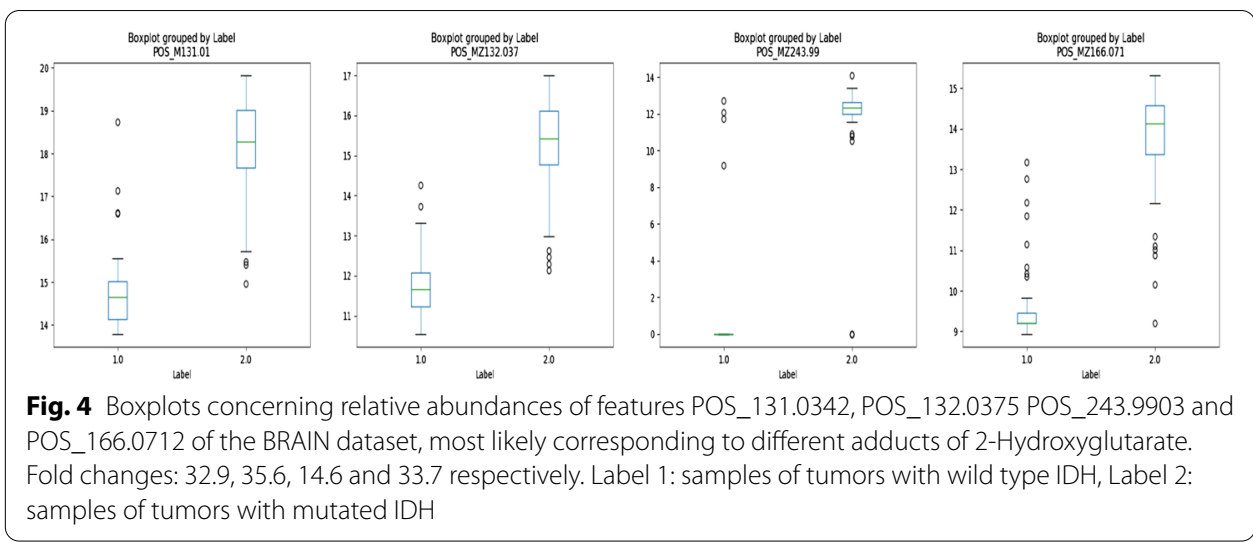

Here all four methods selected this important feature among a high dimensional dataset (25,287 features in this case). Adducts and isotopes of 2-hydroxyglutarate with low levels are top selected features using PD-CR indicating that our method is a very sensitive way to identify significant molecules. This result on the minimally filtered dataset also suggest that PC-CR avoids overfiting as no unexpected feature was selected.

\section{Comparison to the highly filtered dataset}

As shown in Table 6 the accuracies of the different methods were equivalent and very high when using the highly filtered version of the BRAIN dataset (accuracy being a little lower with SVM).

When PD-CR was used on the highly filtered BRAIN dataset, it lead to similar results as with PD-CR using an $\ell 2$ loss, PLS-DA, Random Forests and SVM. In contrast, it outperformed these methods when using the minimally filtered dataset. In this case, as shown in Table 7 more features were selected. When using the BRAIN dataset for the

Table 6 Mean accuracy using 4-fold cross validation with 3 different seeds: comparison of methods on the BRAIN highly filtered data set

\begin{tabular}{llllll}
\hline & PD-CR & PD-CR $\ell_{2}$ & PLS-DA & Random Forests & SVM \\
\hline Accuracy \% & 94.31 & 92.8 & 93.18 & 92.04 & 89.20 \\
\hline
\end{tabular}

Table 7 Top 10 features selected by PD-CR in the highly and minimally filtered versions of the BRAIN dataset

\begin{tabular}{ll}
\hline Identified (495 features) & Large $\mathbf{( 2 5 , 2 8 7}$ features) \\
\hline POS_M131.0342 & POS_MZ131.0342 \\
NEG_M147.02882 & POS_MZ132.0375 \\
POS_M85.0291 & POS_MZ243.9903 \\
POS_M149.0450 & POS_MZ166.0713 \\
NEG_M112.0220 & NEG_MZ147.0288 \\
POS_M154.0864 & NEG_MZ148.0320 \\
NEG_M171.0847 & POS_MZ123.518 \\
NEG_M320.0627 & POS_MZ171.0265 \\
POS_M113.0350 & NEG_MZ149.0329 \\
POS_M147.1170 & POS_MZ133.0384 \\
\hline
\end{tabular}


IDH-mutated vs wild-type classes, most of these additional features were adducts of 2-hydroxyglutarate and are therefore known to be biologically relevant. The additional features that are not adducts of 2-hydroxyglutarate will be investigated in a future study.

\section{Discussion}

Machine learning methods are of particular interest for metabolomics studies and are being used increasingly for other omics studies. Herein we introduce a new primal-dual method for supervised classification and feature selection. To our knowledge, a primaldual method had never been used in this way. We compare this method to three of the most frequently used methods: PLS-DA, Random Forests and SVM, on two metabolomics datasets. Metabolomics datasets tend to be sparse datasets including highly correlated features. PD-CR is particularly suited for this data structure. Hence, for metabolomics, PD-CR appears to be more accurate than the three other methods while selecting biologically relevant features and providing a confidence score for each prediction. An important upside associated with the inclusion of a confidence score for each prediction is that it enables classification with rejection.

We believe that this confidence score is of great value, particularly for applications in medicine. Metabolomics approaches are of particular interest for medical applications. Indeed, they could be used in routine clinical practice as they are relatively inexpensive and can be performed rapidly compared to proteomics, transcriptomics or genomics analyses. More and more studies suggest that metabolomics associated to classification methods are very promising tools for individual personalized medicine [10, 34]. To use metabolomics in routine clinical practice it is paramount to obtain robust, rapid and trustworthy predictions. The confidence score provided with PD-CR adds considerable value to the prediction as it includes a metric that is implicitly used by every physician when they make a medical decision: the probability to make the wrong choice. So far, one of the main obstacles to the use of machine learning in medicine resides in the fact that it is harder to trust the decision of a machine learning method than that of a physician when it comes to health issues. We believe that providing a confidence score associated to the decision would make these new tools more convincing if used in routine clinical practice. Furthermore, this confidence score can be used to perform classification with rejection and reduce the false discovery rate.

Furthermore, this confidence score could be extended to more than 2 classes as follows: We can compute the distances of sample $\mathrm{x}$ to all the centroids, respectively. $d_{1}=\left\|\mu_{i}-x W\right\|_{1}$ and we propose a confidence indicator for sample $\mathrm{x}$ as follows:

$$
\rho(x)=1-k \frac{\operatorname{Min}\left(d_{1}, d_{2} \ldots, d_{k}\right)}{d_{1}+d_{2}+\ldots d_{k}}
$$

Thus, the $\operatorname{CSP} \rho(x)$ is a value ranging from 0 to 1 . The closer the CSP $\rho(x)$ is to +1 for a predicted class, the higher the confidence will be.

We have shown that PD-CR outperformed the common PLS-DA, Random Forests and SVM methods on both LUNG and BRAIN datasets. We believe that this is partly due to the fact that PD-CR uses a Huber loss. Indeed, the use of the Huber loss with PD-CR leads to a better accuracy than the use of a common $\ell 1$ or $\ell 2$ loss [13]. Note that the 11 loss is not derivable in zero. Moreover the drawback of the term $\|Y \mu-X W\|_{1}$ of the 11 
loss is that it enforces equality of the two matrices out of a sparse set. Moreover the use of the Huber loss reduces the impact of the presence of outliers in the training set, and therefore leads to a better accuracy than the $\ell 2$ loss, as shown in Tables 2 and 4 .

Furthermore we show in Tables 2 and 4 that using PD-CR with an $\ell 2$ loss provides better results than PLSDA which uses the same $\ell 2$ loss. This is probably due to the fact that PLS-DA does not perform feature selection and is known to be prone to overfitting [5].

Moreover, when comparing methods with the minimally filtered and the more filtered versions of the BRAIN dataset, all methods suffered a decrease in accuracy with the minimally filtered dataset (PD-CR keeping the higher accuracy). However the results obtained using the PLS-DA method appeared to be more impacted than those of the Random Forests, SVM and PD-CR. Indeed, the accuracy of PLS-DA significantly decreased when the less filtered dataset was used dropping from $93.18 \%$ to $84.09 \%$, compared to a mild decrease in accuracy for the other methods. This can also be explained by the fact that PLS-DA does not perform feature selection and is known to be prone to overfitting [5]. For this reason, several strategies are commonly used to reduce the number of features in metabolomics datasets. Features can be filtered according to the number of detected peaks in all samples, the correct identification of the compound (using the most common adduct) or the presence of isotopes. Working with filtered data has some advantages, including the fact that it appears more biologically relevant to work on less noisy and more reliable data. However, filtering also has some important drawbacks, the most important being the high risk of removing interesting metabolites from the dataset. In the case of the BRAIN dataset, 2-Hydroxyglutarate is a well known metabolite associated to IDH mutation. However, in many metabolomic studies, the goal is to discover potentially unidentified metabolites associated to particular conditions which can only be achieved by including unidentified metabolites. As shown in this work, PD-CR can be applied to both minimally filtered and highly filtered metabolomics datasets.

As it has been previously reported, when designing prediction models, some methods may lead to a more accurate model for a specific dataset while others may be more adapted with other datasets [35]. Indeed, even though we can discuss which machine learning method is the best, most often, researchers try out several machine learning methods on their metabolomics datasets and report the results of the most accurate one. This process has even been automated by some authors [36]. PD-CR is an advanced method, based on recent development in convex optimization and we believe it should be considered by researchers when designing prediction models for metabolomics studies.

Much like the commonly used methods PLS-DA, Random Forests and SVMs, available with [37], our python implementation of PD-CR only requires the tuning of one parameter: $\eta$. This makes the use of PD-CR quite simple, even for non machine learning experts, much like PLS-DA. Note that the tuning of the $\eta$ parameter must be done carefully since it modifies feature selection.

When comparing misclassified patients between methods in an additional analysis, it appeared that in the minimally filtered BRAIN dataset 16/88 tumors were misclassified with at least one method. 2 tumors were misclassified with all methods, 6 with two or three methods and 8 with only one method (3 were misclassfified only with PLS-DA, 
4 with Random Forests, 1 with SVM and none with PD-CR). In the LUNG dataset $702 / 1005$ patients were misclassified with at least one method. 68 patients were misclassified with all methods, 240 with two or three methods and 394 with only one method (15 were misclassfified only with PLS-DA, 63 with Random Forests, 305 with SVM and 11 with PD-CR). It therefore appears that PD-CR is the method with the smallest number of false discoveries.

While prior metabolomic studies did not necessarily focus on validating which features the prediction models relied on, it is now admitted that to be trustworthy a model must be based on biologically relevant features and must therefore be interpretable [38]. Indeed, interpretability of machine learning methods [39] is crucial to assess if selected features are biologically relevant. PD-CR offers a straightforward, reliable metric based on the weights of each feature in the model (matrix W).

Conversely, non-linear methods such as Random Forests or non-linear SVM and the linear methods PLS-DA and linear SVM are usually associated to method-specific metrics which makes it difficult to compare features between methods. For Random Forests, the Mean Decrease Impurity (MDI) is usually the default metric for variable importance [40]. It is computed as a mean of the individual trees' improvement in the splitting criterion produced by each variable. For PLS-DA, the Variable Importance for the Projection (VIP) score is often used. The VIP score is computed by summing the contributions VIN (variable influence) over all model dimensions. For a given PLS dimension $a,(V I N)_{a k}^{2}$ is a function of the squared PLS weight $w_{a k}^{2}$ [41].

While these metrics offer some insight into the importance of each metabolite in the model these are indirect metrics whereas the weights provided with PD-CR represent the direct quantitative measure of the importance of each feature in the model, very close to regression parameters and can thus directly be used to classify a new sample.

Furthermore, relevant feature selection is necessary for a correct understanding of the biological mechanisms underlying classification. It is well established that when expressed, mutant IDH $1 / 2$ reduces 2-oxo-glutarate to 2-hydroxyglutarate [42]. It was therefore expected for 2-hydroxyglutarate to be a feature of importance as was the case when using PD-CR on the BRAIN dataset for the classification of IDH-mutated vs wildtype gliomas. As the biologically relevant features are known in advance, the BRAIN dataset is a good testing set for this new method. Furthermore, as we described, the features selected with PD-CR in the LUNG dataset are identical to the ones described by Mathé et al. in their original study, which also validates the accurate feature selection performed by PD-CR.

\section{Conclusion}

Herein we propose a recently introduced primal-dual method (PD-CR) for feature selection and classification with rejection. To our knowledge, the primal-dual method has never been used in such fashion. PD-CR includes a sparse regularization factor which is particularly appropriate for high dimensional sparse datasets such as metabolomics datasets.

We highlight the two main results. First, PD-CR is more accurate than PLS-DA, Random Forests and SVM and leads to the selection of biologically relevant features. 
Second, our method provides a confidence score for each prediction and allows classification with rejection, which can help reduce false discovery rates.

\section{Supplementary Information}

The online version contains supplementary material available at https://doi.org/10.1186/s12859-021-04478-w.

Additional file 1. Supplementary material: Obtaining metabolomic data for the BRAIN dataset.

\section{Acknowledgements}

The authors thank Xuchun Zhang and Axel Gustovic for his collaboration to the development of python software during their 2018 and 2021 internships respectively, Pr Antonin Chambolle and Pr Jean-Baptiste Caillau for fruitfull discussions concerning the Primal-dual method, Dr Jean-Marie Guigonis (Bernard Rossi facility) for the LC-MS analyses and Pr Hugues Duffau and Dr Catherine Goze for validation, and formal analysis.

\section{Authors' contributions}

DC: Conceptualisation, methodology, formal analysis, investigation, validation, and writing. $\mathrm{OH}$ : funding acquisition, data acquisition and medical evaluation. CB: Data acquisition and medical evaluation. FB-V and VR: Data acquisition and medical evaluation. TP: Conceptualisation, methodology, formal analysis, writing, supervision, resources, project administration, and funding acquisition. MB: Conceptualisation, methodology, formal analysis, writing, supervision, resources, and project administration. All authors read and approved the final manuscript.

\section{Funding}

This work has been supported by the French government, through the UCA-JEDI Investments in the Future project managed by the National Research Agency (ANR) with the reference number ANR-15-IDEX-01. Equipment for this study was purchased through grants from the Recherche en matières de Sûreté Nucléaire et Radioprotection program from the French National Research Agency and the Conseil Départemental 06. David Chardin was funded by a grant from GIRCI Méditéranée.

\section{Availability of data and materials}

We implemented PD-CR in python. Functions and scripts are freely available at https://github.com/tirolab/PD-CR.

\section{Declarations}

\section{Ethics approval and consent to participate}

The samples from the BRAIN dataset were retrospectively collected from two declared biobanks. The authors confirm that all methods were carried out in accordance with relevant guidelines and regulations The authors confirm that informed consent was obtained from all subjects The authors confirm that this retrospective study has been approved by institutional ethics committees of the University Hospital of Nice and of the University Hospital of Montpellier.

\section{Consent for publication}

Not applicalbe.

\section{Competing interests}

The authors declare that they have no competing interests.

\section{Author details}

${ }^{1}$ Transporters in imaging and Radiotherapy in Oncology (TIRO), Direction de la Recherche Fondamentale (DRF), Institute des sciences du vivant Fréderic Joliot, Commissariat à l'Energie Atomique et aux énergies alternatives (CEA), Université Côte d'Azur (UCA), Nice, France. ${ }^{2}$ Department of Nuclear Medicine, Centre Antoine Lacassagne, Université Côte d'Azur (UCA), Nice, France. ${ }^{3}$ Laboratoire d'Informatique, Signaux et Systèmes de Sophia Antipolis (I3S), Université Côte d'Azur (UCA), Centre de Recherche Scientifique (CNRS), Sophia Antipolis, France. ${ }^{4}$ Department of Oncology, Centre Antoine Lacassagne, Université Côte d'Azur (UCA), Nice, France. ${ }^{5}$ Central Laboratory of Pathology, University Hospital and Institute of Biology Valrose, Inserm U1091 - CNRS UMR7277, University Côte d'Azur, Nice, France. ${ }^{6}$ Department of Pathology and Oncobiology, University Hospital, Montpellier, France. ${ }^{7}$ Institute for Neurosciences of Montpellier, INSERM U1051, Montpellier, France.

Received: 19 January 2021 Accepted: 29 October 2021

Published online: 15 December 2021

\section{References}

1. Johnson CH, Ivanisevic J, Siuzdak G. Metabolomics: beyond biomarkers and towards mechanisms. Nat Rev Mol Cell Biol. 2016;17(7):451-9. https://doi.org/10.1038/nrm.2016.25.

2. Kell DB. Metabolomics and systems biology: making sense of the soup. Curr Opin Microbiol. 2004;7(3):296-307. https://doi.org/10.1016/j.mib.2004.04.012.

3. Barker M, Rayens W. Partial least squares for discrimination. J Chemom. 2003;17(3):166-73. 
4. Gromski PS, Muhamadali H, Ellis DI, Xu Y, Correa E, Turner ML, Goodacre R. A tutorial review: Metabolomics and partial least squares-discriminant analysis - a marriage of convenience or a shotgun wedding. Anal Chim Acta. 2015;879:10-23. https://doi.org/10.1016/j.aca.2015.02.012.

5. Westerhuis JA, Hoefsloot HCJ, Smit S, Vis DJ, Smilde AK, van Velzen EJJ, van Duijnhoven JPM, van Dorsten FA. Assessment of PLSDA cross validation. Metabolomics. 2008;4(1):81-9. https://doi.org/10.1007/ s11306-007-0099-6.

6. Brereton RG. Consequences of sample size, variable selection, and model validation and optimisation, for predicting classification ability from analytical data. TrAC, Trends Anal Chem. 2006;25(11):1103-11. https://doi.org/ 10.1016/j.trac.2006.10.005.

7. Broadhurst DI, Kell DB. Statistical strategies for avoiding false discoveries in metabolomics and related experiments. Metabolomics. 2006;2(4):171-96. https://doi.org/10.1007/s11306-006-0037-z.

8. Bartel J, Krumsiek J, Theis FJ. Statistical methods for the analysis of high-throughput metabolomics data. Comput Struct Biotechnol J. 2013;4(5):201301009. https://doi.org/10.5936/csbj.201301009.

9. Breiman L. Random forests. Mach Learn. 2001;45(1):5-32.

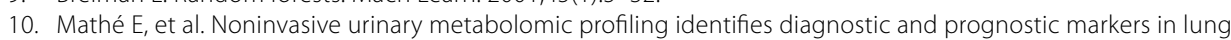
cancer. Cancer Res. 2014;74(12):3259-70.

11. Heinemann J, Mazurie A, Tokmina-Lukaszewska M, Beilman GJ, Bothner B. Application of support vector machines to metabolomics experiments with limited replicates. Metabolomics. 2014;10(6):1121-8. https://doi. org/10.1007/s11306-014-0651-0.

12. Guyon I, Weston J, Barnhill S, Vapnik V. Gene selection for cancer classification using support vector machines. Mach Learn. 2002;46(1-3):389-422.

13. Barlaud M, Chambolle A, Caillau J-B. Classification and feature selection using a primal-dual method and projection on structured constraints. International Conference on Pattern Recognition, Milan, 2020;6538-6545.

14. Barlaud M, Chambolle A, Caillau J-B. Classification and feature selection using a primal-dual method and projection on structured constraints. In: International Conference on Pattern Recognition, Milan, 2020;6538-6545.

15. Jacob L, Obozinski G, Vert J-P. Group lasso with overlap and graph lasso. In: Proceedings of the 26th International Conference on Machine Learning (ICML-09), 2009;353-360.

16. Ding C, Li T. Adaptive dimension reduction using discriminant analysis and k-means clustering. In: Proceedings of the 24th International Conference on Machine Learning, 2007;521-528.

17. Combettes J-C PL, Pesquet. A douglas-rachford splitting approach to nonsmooth convex variational signal recovery. IEEE J. Selected Topics Signal Process., 2007;564-574.

18. Barlaud M, Belhajali W, Combettes PL, Fillatre L. Classification and regression using an outer approximation projection-gradient method. IEEE Trans Signal Process. 2017:65(17):4635-44.

19. Freund MYY, Schapire RE. Generalization bounds for averaged classifiers. Ann Stat. 2004;32(4):1698-722.

20. Nock R, BelHajAli W, Dambrosio R, Nielsen F, Barlaud M. Gentle nearest neighbors boosting over proper scoring rules. IEEE. 2015;37:80-93.

21. Hastie T, Rosset S, Tibshirani R, Zhu J. The entire regularization path for the support vector machine. J Mach Learn Res. 2004;5:1391-415.

22. Friedman J, Hastie T, Tibshirani R. Regularization path for generalized linear models via coordinate descent. J Stat Softw. 2010;33:1-122.

23. Hastie T, Tibshirani R, Wainwright M. Statistcal learning with sparsity: The lasso and generalizations. CRC Press;2015.

24. Li J, Cheng K, Wang S, Morstatter F, P Trevino R, Tang J, Liu H. Feature selection: A data perspective. ACM Computing Surveys 2016:50.

25. Ali A, Tibshirani R. The generalized lasso problem and uniqueness. Electron J Stat. 2019;13(2):2307-47.

26. McQueen J-B. Some methods for classification and analysis of multivariate observations. In: Proceedings of the fifth Berkeley Symposium on Mathematical Statistics and Probability; 1967.

27. Chambolle A, Pock T. On the ergodic convergence rates of a first-order primal-dual algorithm. Math Program. 2016;159(1-2):253-87.

28. $\mathrm{Ni} \mathrm{C}$, Charoenphakdee N, Honda J, Sugiyama M. On the calibration of multiclass classification with rejection;2019. arxiv:1901.10655.

29. Louis DN, Perry A, Reifenberger G, von Deimling A, Figarella-Branger D, Cavenee WK, Ohgaki H, Wiestler OD, Kleihues P, Ellison DW. The 2016 world health organization classification of tumors of the central nervous system: a summary. Acta Neuropathol. 2016;131(6):803-20. https://doi.org/10.1007/s00401-016-1545-1.

30. Yoshida A, Satomi K, Ohno M, Matsushita Y, Takahashi M, Miyakita Y, Hiraoka N, Narita Y, Ichimura K. Frequent falsenegative immunohistochemical staining with IDH1 (R132H)-specific H09 antibody on frozen section control slides: a potential pitfall in glioma diagnosis. Histopathology. 2019;74(2):350-4. https://doi.org/10.1111/his.13756.

31. van den Berg RA, Hoefsloot HCJ, Westerhuis JA, Smilde AK, van der Werf MJ. Centering, scaling, and transformations: improving the biological information content of metabolomics data. BMC genomics 2006;7.

32. Wold S, Sjostrom M, Eriksson L. PIs-regression: a basic tool of chemometrics. Elsevier. 2001;58(2):109-30.

33. Dang L, White DW, Gross S, Bennett BD, Bittinger MA, Driggers EM, Fantin VR, Jang HG, Jin S, Keenan MC, Marks KM, Prins RM, Ward PS, Yen KE, Liau LM, Rabinowitz JD, Cantley LC, Thompson CB, Vander Heiden MG, Su SM. Cancerassociated IDH1 mutations produce 2-hydroxyglutarate. Nature. 2009;462(7274):739-44. https://doi.org/10.1038/ nature08617.

34. Jing L, Guigonis J-M, Borchiellini D, Durand M, Pourcher T, Ambrosetti D. LC-MS based metabolomic profiling for renal cell carcinoma histologic subtypes. Sci Rep. 2019:9(1):1-10.

35. Madsen R, Lundstedt T, Trygg J. Chemometrics in metabolomics-A review in human disease diagnosis. Anal Chim Acta. 2010;659(1):23-33. https://doi.org/10.1016/.aca.2009.11.042

36. Leclercq M, Vittrant B, Martin-Magniette ML, Scott Boyer MP, Perin O, Bergeron A, Fradet Y, Droit A. Large-scale automatic feature selection for biomarker discovery in high-dimensional OMICs data. Front Genet. 2019. https://doi.org/ 10.3389/fgene.2019.00452. 
37. Xia J, Psychogios N, Young N, Wishart DS. MetaboAnalyst: a web server for metabolomic data analysis and interpretation. Nucleic Acids Res 37(suppl 2), 2009;652-660. https://doi.org/10.1093/nar/gkp356. https://academic.oup.com/ nar/article-pdf/37/suppl_2/W652/3933058/gkp356.pdf.

38. Zhang A, Sun H, Yan G, Wang P, Wang X. Mass spectrometry-based metabolomics: applications to biomarker and metabolic pathway research. Biomed Chromatogr. 2016;30(1):7-12.https://doi.org/10.1002/bmc.3453. https://onlin elibrary.wiley.com/doi/pdf/10.1002/bmc.3453. Accessed 2020-05-13.

39. Doshi-Velez F, Kim B. Towards a rigorous science of interpretable machine learning (2017). arxiv:1702.08608.

40. Pedregosa F, Varoquaux G, Gramfort A, Michel V, Thirion B, Grisel O, Blondel M, Müller A, Nothman J, Louppe G, Prettenhofer P, Weiss R, Dubourg V, Vanderplas J, Passos A, Cournapeau D, Brucher M, Perrot M, Duchesnay Ã. Scikitlearn: Machine Learning in Python. arXiv:1201.0490 [cs] (2018). arXiv: 1201.0490. Accessed 2020-05-11.

41. Chong I-G, Jun C-H. Performance of some variable selection methods when multicollinearity is present. Chemom Intell Lab Syst. 2005;78(1):103-12. https://doi.org/10.1016/j.chemolab.2004.12.011.

42. Losman J-A, Kaelin WG. What a difference a hydroxyl makes: mutant IDH, (R)-2-hydroxyglutarate, and cancer. Genes Dev. 2013;27(8):836-52. https://doi.org/10.1101/gad.217406.113Company: Cold Spring Harbor Laboratory Press Distributor: Cold Spring Harbor Laboratory Press Institution: Cold Spring Harbor Laboratory Press Label: Cold Spring Harbor Laboratory Press Publisher: Cold Spring Harbor Lab. Accessed 2020-05-19.

\section{Publisher's Note}

Springer Nature remains neutral with regard to jurisdictional claims in published maps and institutional affiliations.

- fast, convenient online submission

- thorough peer review by experienced researchers in your field

- rapid publication on acceptance

- support for research data, including large and complex data types

- gold Open Access which fosters wider collaboration and increased citations

- maximum visibility for your research: over 100M website views per year

At BMC, research is always in progress.

Learn more biomedcentral.com/submissions 